\title{
Cosmic Ray Breakdown Mechanism in the Atmophere
}

\author{
Akande, P. $\mathrm{I}^{[1]}$, Azi Amaechi O. ${ }^{[2]}$, A. O. Chikwendu ${ }^{[3]}$, Nwoba Isreal A. ${ }^{[4]}$ \\ ${ }^{[1][2]}$ Department of Industrial Physics, Ebonyi State University, Abakaliki \\ ${ }^{[3]}$ Department of Industrial Physics, Renaissance University, Ugbawka \\ ${ }^{[4]}$ Evangel University, Akaeze, PMB 126, Ebonyi State
}

\begin{abstract}
Cosmic rays are high energy particles originating from outer space. These cosmic rays produce secondary cosmic rays as they enter the atmosphere and generate runaway electrons. The generation of runaway electrons in the relativistic region of the atmosphere is the focus of this work. To achieve our aim, basic consideration is given to the cold electron having mean velocity which is less than electron thermal speed. The effects of force on these electrons are obtained. From the result, it shows that cosmic ray breakdown occurs in the atmosphere within the relativistic region and the electrons are accelerated by the electric field. Hence there is an exponential increase of the fast electrons. This exponential increase in fast electron in the atmosphere could be the basic reason for the spontanouse characteristic behavour of most atmospheric processes.
\end{abstract}

\section{Introduction}

Most cosmic rays are thought to originate outside the solar system, with many coming from within our Milky Way Galaxy, and a few arriving from other galaxies, (James, 1998). This cosmic ray produce secondary particle which can be sufficiently energetic to contribute themselves to further ionization of the neutral gases. Due to their charges, cosmic ray particles are additionally deflected by the geomagnetic field. Almost all particles can penetrate into the polar region, where the magnetic field lines are perpendicular to the ground, where as only the most highly energetic electron particles of these cosmic rays with energy above $15 \mathrm{Gev}$ are able to penetrate the lower atmosphere near the equator, (Devendra and Singh,2010). Such energetic electrons are present in the atmosphere as secondaries generated by cosmic rays electron accelerated in fully ionized atmosphere. However, cosmic rays are the only source of ion production in the lower atmosphere which is confirmed from the measurements carried out by Ermakov and Komotskov (Ermakov et al, 1992). Therefore, the processes, depending on the electrical properties of the atmosphere such as atmospheric electric current, lightening production, cloud and thunder cloud formation etc, can be affected by cosmic rays. For studying cosmic ray ground based observatories covering large areas are needed because the flux of cosmic rays gaze too low at these energies for direct measurement by balloons or satellite based experiment (Apel and Arteage, 2011).

The cosmic rays exert much influence on the Earth's atmosphere since many atmospheric processes (e.g. atmospheric electric current, cloud and thundercloud formation, concentration of heavy and light ion) are defined by their electrical properties (Harrison and Stephenson , 2006).

At certain altitudes, cosmic rays are believed to be a source of atmospheric ionization and could be responsible for electrical conductivity of the atmosphere at lower altitudes. It has been reported that ion production rate in the atmosphere and cosmic ray intensity variations could account for atmospheric current and lightning occurrence at middle latitudes. Wilson, (1924) suggested that thunderstorm electric field could accelerate cosmic ray secondary electrons to high energies (Wilson, 1924). The showers of energetic particles produced by such high energy cosmic rays could provide a conducting path that initiates lightning (Gurevich et al, 1999). In nature, runaway phenomena occur in lightning path and a variety of astrophysical situations (Wilson, 1925). Charge separation in a thundercloud results in buildup of an electric field between the top and bottom. One might expect that the breakdown potential for lightning in a thundercloud is close to the typical potential measured in air, but investigations have revealed a much lower breakdown potential (Gurevich, 1992; Aleksandr et al, 2009). This occurs due to the above avalanche electron in which low-energy electrons are knocked into runaway by high-energy electrons, with the original high-energy electrons presumably generated by a cosmic ray or radioactive decay of an atmospheric atom. Such high-energy electrons can have a mean free path of a few kilometers over which they gain even more energy.

\section{Methodology}

In this work, consideration is given to the secondary electrons generated by cosmic rays which are influenced by the electric field in the atmosphere. If cold electron has mean velocity $v$ less than the electron thermal speed $v_{t}$, it will undergo a dynamical frictional force, and according to Milikh is given by; 
$F=m V_{o} v$

this will be used to derive the equation which shows the rate of electron breakdown in the atmosphere within the relativistic region.

From equation (1),

\section{Result}

$V_{o}=\frac{4 \pi e^{4} Z N_{m}}{m^{2} v^{3}} \log _{e} \Lambda$

If we consider a fast electron having larger velocity with its dynamical frictional force reduced when the velocity increases, the force will be

$F=\frac{4 \pi e^{4} Z N_{m}}{m c^{2}} \log _{e} \Lambda$

Where $\log _{e} \Lambda=\log _{e}\left\{\left(\frac{m c^{2}}{\epsilon_{1}}\right) \gamma\right\}, \gamma$ is the Lorentz factor.

$F=\frac{4 \pi e^{4} Z N_{m}}{m c^{2}} \log _{e}\left\{\left(\frac{m c^{2}}{\epsilon_{1}}\right) \gamma\right\}$

$\log _{e}\left\{\left(\frac{m c^{2}}{\epsilon_{1}}\right) \gamma\right\}=F \div \frac{4 \pi e^{4} Z N_{m}}{m c^{2}}$

But electric field intensity, $E=\frac{F}{e}$

Therefore,

$\log _{e}\left\{\left(\frac{m c^{2}}{\epsilon_{1}}\right) \gamma\right\}=E e \div \frac{4 \pi e^{4} Z N_{m}}{m c^{2}}$

$\log _{e}\left\{\left(\frac{m c^{2}}{\epsilon_{1}}\right) \gamma\right\}=\frac{E e}{1} \times \frac{m c^{2}}{4 \pi e^{4} Z N_{m}}$

$\log _{e}\left\{\left(\frac{m c^{2}}{\epsilon_{1}}\right) \gamma\right\}=\frac{E m c^{2}}{4 \pi e^{3} Z N_{m}}$

$\log _{e}\left\{\left(\frac{m c^{2}}{\epsilon_{1}}\right) \gamma\right\}=\frac{E m c^{2} a}{4 \pi e^{3} Z N_{m} a}$

Let a dimensionless parameter $\delta_{o}=\frac{E m c^{2} a}{4 \pi e^{3} Z N_{m} a}$

$\log _{e}\left\{\left(\frac{m c^{2}}{\epsilon_{1}}\right) \gamma\right\}=\delta_{o} a$

$\left(\frac{m c^{2}}{\epsilon_{1}}\right)+\log _{e} \gamma=\delta_{o} a$

$a+\log _{e} \gamma=\delta_{o} a$

$\gamma=\operatorname{Exp}\left\{a\left(\delta_{o}-1\right)\right\}$

This shows that the electrons are accelerated by the electric field.

\section{Discussion And Conclusion}

From the result, in equation (2), the frictional force opposes the motion of electrons moving in a distribution of field particles. And as the frictional force tends to the relativistic factor, in equation (3) it will decrease gradually. The introduction of a dimensionless parameter is to show that the electric field is larger than minimum. This is because, when the magnitude of the frictional force is minimum, the electrons move relativistically, which implies that some of the cosmic ray electrons have energy higher than the critical field and in turn are able to generate fast electrons. In equation (6), it shows that the production of these fast electrons is accompanied by an exponential increase in the number of thermal secondary electrons. This is in agreement with Gurevich et al (1992). That is, the is an electrical breakdown of gas. This describes a mechanism for air breakdown in which high energy electrons are accelerated by electric fields to relativistic energies. This is in comparison with Tierney,et al. Hence there is an exponential increase of the fast electrons. This exponential increase in fast electron in the atmosphere could be the basic reason for the spontanouse characteristic behavour of lightening production and the occurrences of other atmospheric perturbation. 


\section{References}

[1]. Aleksandr, V., Gurevich, A. N., Vladimir, A. R., Aleksandr, P. C. and Aleksandr, L. S. (2009). Non-linear Phenomena in the Ionospheric Plasma: Effects of Cosmic Rays and Runaway Breakdown on Thunderstorm Discharges. Ann Geophys, 24, 529542.

[2]. Apel, W.D and Arteage J.C,(2011). Thunderstorm observations by air-shower radio antenna arrays. Advances in space Research (a cospar publication). Vol.48, pp 1295-1303.

[3]. Devendra Simgh and Singh R.P, (2010). The role of cosmic rays in the Earth's atmospheric processes. Centre for sun-climate Research, Danish National space Institute copehagen, Denmark. Vol. 74. Pp 153. 168.

[4]. Ermakov, V.I., Kokin, G.A., Komotskov, A.V., Sorokin,M.G., (1992). Results of measurements of the concentrationof negative ions in the polar stratosphere Geomagnetismand Aeronomy 32 (3), 47.

[5]. Gurevich, A.V; Milikh G.M; and Roussel Dupre R. (1992).Runaway electron mechanism of air breakdown and preconditioning during a thunderstorms, physics letters A, vol. 165,468.

[6]. Gurevich, A. V; Zybin K. P. and Roussel- Dupre, R. A, (1999). Lighterning initiation by simultaneous effect of runaway breakdown and cosmic ray showers physics Lectures A, 254: 79-87.

[7]. Harrison, R. D and Stephenson D. B., (2006). Detection of a galactic cosmic ray influence on clouds, Geophysical Research Abstracts, vol.8, $\quad 07661$

[8]. James, W. Cronin and Thomas. K. Gaisser and simon. P. Swordy (1998). Scientific American.

[9]. Tierney, H. E., R. A. Roussel-Dupré, and E. M. D. Symbalisty. "NUMERICAL SIMULATIONSOFHIGH-ENERGY ELECTRON AVALANCHES IN THUNDERSTORM ELECTRIC FIELDS AND ASSOCIATED RADIO

EMISSIONS."

[10]. Wilson, CT. R., (1924). The electric field of a thundercloud and some of its effects. Proc.

[11]. Wilson, C.T. R., (1925). The acceleration of particles in strong electric fields such

as those

Phys. Soc.London. 37: 320 Mathematical Proceedings of the Cambridge Philosophical Society, 22(04):534-538. 